\title{
Withholding and withdrawal of life support in critically ill patients at the end of life: pain is not the only symptom to manage
}

\author{
Lindsey Obarzanek * (1) and Margaret L. Campbell
}

(C) 2021 Springer-Verlag GmbH Germany, part of Springer Nature

We read with interest the recent article presenting a systematic review and meta-analysis summarizing pharmacological pain management during withholding as well as withdrawal of life support for critically ill patients at end-of-life [1]. We acknowledge the importance of this review and its contribution to end-of-life research as well as clinical management of this vulnerable population.

The authors' comprehensive use of Cochrane methodology and the Preferred Reporting Items for Systematic Reviews and Meta-Analyses (PRISMA) provided desired clarity in the structure of the review process. We value the authors' dedication to the ongoing review of this important topic; however, this study's inclusion criteria and organization left us questioning the clinical applicability given the single symptom focus in two highly variable clinical situations.

Pain was the primary focus of the article, as it is the most frequently reported distressing symptom in patients at high risk of dying whom are able to self-report [2]. Pain management is often the primary focus in achieving comfort, but overall comfort is multi-dimensional and can encompass the patient's subjective experience of numerous symptoms. Pain is well-studied and deserves ongoing attention; however, we feel that it is equally important to examine and treat what has been reported as the most distressing symptom, which is dyspnea [2]. Dyspnea and its observed signs are expected in patients who opt for no ventilation, and particularly for ventilator-dependent patients undergoing withdrawal. Dyspnea can and should be measured and treated in both self-reporting and nonself-reporting patients, especially at the end-of-life [3].

*Correspondence: AL2569@wayne.edu

College of Nursing, Wayne State University, Detroit, MI, USA
Dyspnea is often bundled in evaluation of overall comfort, but should be examined and treated individually in this time of focused care and symptom science.

Withholding ventilator support and withdrawal of ventilator support are two different end-of-life processes for patients. The non-pharmacological and pharmacological needs to maintain comfort, while withholding intubation vs. withdrawing ventilation seem difficult to collapse into one area of study. The individualized symptoms which patients experience may vary in these two separate treatment plans. For example, patients opting to withhold ventilation may be treated with non-invasive ventilation or high-flow nasal oxygen [4]. Patients undergoing withdrawal are typically treated with morphine or fentanyl in response to signs of respiratory distress. These specific instances in patient care may be best examined in separate cases, as they oppose each other in the trajectory of care.

In addition, we expect to see what measures were used to assess discomfort, as well as what was the appropriate criteria for achieving pain control [5]. In Table 1, the "Outcomes used in this review" column predominantly reports sedatives and analgesics, not patient-reported or patient-observed outcomes. In the few articles where pain was examined, the specific measure was not identified, nor was how to determine adequate pain relief. As clarity of methods is critical for study replication to further advance science, the lack of specificity appeared vague when evaluating critical study factors.

With increased symptom focus and outcome clarity, it is hopeful that studies such as this can further advance our end-of-life care and lead to optimal comfort outcomes for the described patients. 


\section{Compliance with ethical standards}

\section{Conflict of interest}

The authors have no conflict of interest with this submission.

\section{Publisher's Note}

Springer Nature remains neutral with regard to jurisdictional claims in published maps and institutional affiliations.

Accepted: 30 December 2020

Published online: 11 February 2021

\section{References}

1. Laserna A, Durán-Crane A, López-Olivio MA et al (2020) Pain management during the withholding and withdrawal of life support in critically ill patients at the end-of-life: a systematic review and meta-analysis. Intensive Care Med 46:1671-1682. https://doi.org/10.1007/s00134-020-06139 $-7$

2. Puntillo KA, Aria S, Cohen NH et al (2010) Symptoms experienced by intensive care unit patients at high risk of dying. Crit Care Med 38(11):2155-2160. https://doi.org/10.1097/CCM.0b013e3181f267ee

3. Campbell ML, Templin T, Walch J (2010) A respiratory distress observation scale for patients unable to self-report dyspnea. J Pall Med 13(3):285-290. https://doi.org/10.4037/15597768-2007-4008

4. Nava S, Ferrer M, Esquinas A et al (2013) Palliative use of non-invasive ventilation in end-of-life patients with solid tumours: a randomised feasibility trial. Lancet Oncol 14(3):219-227. https://doi.org/10.1016/S1470 -2045(13)70009-3

5. Gelinas C, Fillion L, Puntillo KA, Viens C, Fortier M (2006) Validation of the critical-care pain observation tool in adult patients. Am J Crit Care Off Pub Am Assoc Critical-Care Nurses 15(4):420-427. https://doi.org/10.4037/ AJCC2006.15.4.420 